Erbschaftsteuer

\section{Chancengerechtigkeit erhöhen}

Die persönliche Vermögensteuer unterscheidet nicht, ob das belastete Vermögen selbst verdient oder den Zufällen des Erbens geschuldet ist. Derjenige, der sein Vermögen durch langjährige Erwerbsarbeit und Konsumverzicht gebildet hat, wird genauso zur Kasse gebeten wie derjenige, der nie gearbeitet hat und dem ein Vermögen in gleicher Höhe in den Schoß gefallen ist. Dasselbe gilt für die Besteuerung der Vermögenseinkommen. Im Gegensatz zu diesen beiden Steuern belastet die Erbschaftsteuer zielgenau das leistungslose Vermögen. Sie ist das einzige steuerliche Instrument, das dem Gemeinwesen zur Verfügung steht, um die elementare Chancenungerechtigkeit zu korrigieren, die sich daraus ergibt, dass einige von besitzlosen und andere von besitzstarken Eltern erben.

Aber sind Erbschaften ökonomisch so relevant, dass sie eine solche Besteuerung in der Praxis rechtfertigen? Zwei empirische Befunde heben die ökonomische Bedeutung der Erbschaftsteuer hervor. Zum einen sind Erbschaften unter Individuen der gleichen Kohorte sehr ungleichmäßig verteilt. Viele Individuen erben in ihrem gesamten Leben sehr wenig oder überhaupt nichts; eine kleine Minderheit erbt Vermögensvolumina, die das durchschnittliche Lebenserwerbseinkommen der Bevölkerung um ein Vielfaches übersteigen. In Deutschland drückt sich diese Ungleichheit unter anderem in einem ausgeprägten regionalen Gefälle aus: Der mittlere Wert einer Erbschaft ist in Ostdeutschland weniger als halb so hoch wie in Westdeutschland. Eine Erbschaftsteuer, die tatsächlich progressiv ist, kann diesen Missstand maßgeblich begrenzen. Zum anderen beobachtet man in Deutschland und anderen entwickelten Volkswirtschaften, dass das gesamtwirtschaftliche jährliche Erbschaftsvolumen in Relation zum BIP permanent steigt. Diese Entwicklung deutet auf ein nicht zu vernachlässigendes steuerliches Aufkommenspotenzial hin, das eine Regierung nutzen könnte, um diejenigen zu kompensieren, denen sich die Geburtslotterie als weniger gnädig gezeigt hat. Die Erbschaftsteuer ist somit keine Nebensache und ihre Abschaffung hätte weitreichende langfristige soziale Kosten zur Folge. Umgekehrt kann eine Erbschaftsteuer ein von praktisch allen Bürger:innen geteiltes Ideal von Chancengerechtigkeit zum Ausdruck bringen und gleichzeitig die sozioökonomische Mobilität und den persönlichen Verdienst fördern. Ferner kann eine Erbschaftsteuer die Luft aus ökonomisch abwegigen, aber politisch aussichtsreichen Vorschlägen

(C) Der/die Autor:in(nen) 2021. Open Access: Dieser Artikel wird unter der Creative Commons Namensnennung 4.0 International Lizenz veröffentlicht (creativecommons.org/licenses/by/4.0/deed.de).

Open Access wird durch die ZBW - Leibniz-Informationszentrum Wirtschaft gefördert. herausnehmen - wie etwa der Wiedereinführung der Vermögensteuer in Deutschland -, die durch ein Defizit an sozialer Gerechtigkeit fortwährend genährt werden. Benötigt wird eine ethisch und ökonomisch glaubwürdige Steuer. Diese Eigenschaften besitzt die derzeitige Besteuerung von Erbschaften und Schenkungen in Deutschland nicht.

Erstens sollte die Bemessungsgrundlage der Erbschaftsteuer möglichst breit sein; sie sollte alle Bestandteile des geerbten Vermögens gleich behandeln und vorsichtige Bewertungsverfahren beinhalten. Dennoch gibt es einen Elefanten im Raum: Die derzeitige faktische Steuerbefreiung des Betriebsvermögens, die genau die Empfänger:innen der größten Erbschaften aus dieser Verpflichtung des Eigentums nimmt. Diese Freistellung wird unter Bedingungen gewährt, die vermeintlich positive arbeitsmarktpolitische Effekte haben sollen. Sie ist nicht nur deswegen schädlich, weil die entsprechenden Vorschriften gravierende ökonomische Fehlanreize stiften, sondern auch weil sie eine herbe Ohrfeige gegen die Prinzipien von Fairness, sozialer Mobilität und Förderung individueller Leistungsbereitschaft sind.

Zweitens und unter der Voraussetzung, dass das geerbte Betriebsvermögen ohne Ausnahmeregelung in die Bemessungsgrundlage integriert wird, sollten die Freibeträge erhöht und die Steuersätze verringert werden, um eine sanfte Transition zu einer vernünftigen Besteuerung zu schaffen. Der von Lobbys immer wieder hervorgebrachten Mär der Insolvenz von Betrieben infolge der Erbschaftbesteuerung fehlt jegliche empirische Grundlage. Auch für die gemahnten Auswanderungseffekte gibt es keine empirische Evidenz. Sogar unter den Schweizer Kantonen scheint die unterschiedliche Höhe der Erbschaftsteuer keine signifikante Relokalisation von Steuerpflichtigen und ihren wirtschaftlichen Aktivitäten in Gang zu setzen. Gleichwohl wäre in Deutschland die steuerliche Gleichbehandlung vom Betriebsvermögen eine substanzielle Verschärfung im Vergleich zum Status quo, und ihre Anreizwirkung kann nicht präzise genug vorausgesehen werden.

Und wenn die Gesetzgebung der Stimme der Vernunft folgen will, sollte sie die Gelegenheit nicht verpassen, den derzeitigen Tariftypus durch den üblichen Anstoßtarif zu ersetzen, bei dem der Grenzsteuersatz stufenweise steigt. Auf diese Weise würde sich das Problem der Rangumkehr, das vom jetzigen Tarif hervorgerufen wird, erübrigen. Bei der Erbschaftsteuer hat man in Deutschland den Eindruck, dass die Gesetzgebung bisher nichts unterlassen hat, was das Bundesverfassungsgericht veranlassen könnte, dieser Steuer das Aus zu bereiten. Möge ein Sinneswandel eintreten.

Giacomo Corneo

Freie Universität Berlin

giacomo.corneo@fu-berlin.de 